\title{
EDITORIAL
}

\section{Diabetes, detección temprana para una enfermedad compleja}

$\mathrm{G}$ lobalmente, la diabetes es considerada como una enfermedad casi pandémica por su alta prevalencia y crecimiento progresivo; en las últimas cuatro décadas ha incrementado del 4,3\% al 9,0\% en hombres y del 5,0\% al 7,9\% en mujeres ${ }^{(1)}$. Sin embargo, sabemos que el número de personas diagnosticadas con diabetes en Colombia es muy bajo, comparado con las cifras reales esperadas en nuestro país, así como en el mundo.

La Encuesta Nacional de Demografía y Salud 2010 (ENDS 2010) proporcionó datos de personas mayores de 60 años diagnosticadas con diabetes según el grupo de edad: 60-64 años, 9,8\%; 65-69 años, 10,8\%; 70-74 años, 13\%; $75-79$ años, $12,4 \%$; 80-84 años, 13,1\%; 85-89 años, 7,5\%; 90-94 años, 9,5\%; y >95 años, $11,7 \%{ }^{(2)}$. El porcentaje nacional total fue de $11,2 \%$ y en Guainía fue del $16,7 \%^{(2)}$. Como vemos, en nuestro país la prevalencia varía, dependiendo de las regiones estudiadas, del rango poblacional evaluado, y de otra serie de variables que influyen, como: sexo, profesión, criterios diagnósticos utilizados y comorbilidades asociadas según los estudios previamente publicados.

El comportamiento epidemiológico de la diabetes en Colombia se ve afectado por varias circunstancias propias de nuestra región como: transición demográfica (envejecimiento poblacional), transición nutricional, desplazamiento forzado, fenómeno migratorio, urbanización, crecimiento económico, nivel educativo, vulnerabilidad poblacional, así como promoción de la actividad física ${ }^{(3)}$.

La diabetes en Colombia es una de las cinco primeras causas de muerte y está entre las diez primeras causas de consulta en adultos, por lo cual es un problema de salud pública que debe tener estrategias de prevención en todas sus etapas, empezando por un diagnóstico temprano.

La identificación de los individuos con diabetes no diagnosticada, por medio de una escala de riesgo como el FINDRISC, puede ser una importante aproximación para prevenir o retardar complicaciones de la diabetes tipo 2. El FINDRISC es un cuestionario originalmente creado para realizarlo en las personas entre los 35 y 64 años, que ayuda a identificar a individuos en alto riesgo de desarrollar la enfermedad ${ }^{(4)}$. Este método de tamizaje ha sido validado en diferentes países y en Colombia, recomendándose en las Guías Nacionales como el método de tamización adaptado para la población colombiana, para detectar individuos con alto riesgo de diabetes tipo 2 en adultos y que tengan un puntaje $\geq 12^{(5)}$; esto permite que se pueda educar a las personas en mayor riesgo, pero que aún no son diabéticas, beneficiándolas de la inclusión en programas de prevención con nueva tamización a los tres años ${ }^{(5)}$.

Al aplicar el FINDRISC colombiano, además de los factores de riesgo identificados, es probable que influyan otros para los nuevos casos de diabetes o para que ésta se desarrolle, como son los factores medioambientales y socioeconómicos. El envejecimiento de nuestra población, así como en Suramérica, aportará un número importante de individuos con diabetes con consecuencias negativas desde el punto de vista social y económico ${ }^{(6)}$.

La obesidad y el sobrepeso son factores que influyen en la frecuencia de prediabetes y diabetes y la prevalencia está incrementando en nuestra población. En algunas regiones existe un mayor número de personas con esta connotación y además está ligada a los otros factores mencionados.

Es muy importante que, teniendo la herramienta para tamizar, se realicen estudios poblacionales en todo el país y se logre identificar un número de diabéticos más cercano a la realidad y se instalen e intensifiquen medidas de salud pública, tanto de prevención como de manejo adecuado para una enfermedad epidémica como el caso de la diabetes.

\section{Luz Ángela Casas}

Médica Endocrinóloga, FACE

Jefe sección de Endocrinología Fundación Valle del Lili

Profesora Universidad ICESI

Expresidente y Miembro Honorario de la Asociación Colombiana de Endocrinología, Diabetes y Metabolismo

Miembro del Comité Editorial de la Revista Colombiana de Endocrinología, Diabetes y Metabolismo

\section{Referencias}

1. NCD Risk Factor Collaboration (N.C.D.RisC), Worldwide trends in diabetes since 1980: a pooled analysis of 751 population-based studies with 4.4 million participants, Lancet 387 (2016) 1513-1530.

2. Encuesta Nacional de Demografía y Salud - ENDS. Available at: http://www.profamilia.org.co/encuestas/Profamilia/ Accessed October 25, 2015.

3. Vargas H, Casas L. A, An Epidemiologic Analysis of Diabetes in Colombia, Annals of Global Health, Vol 81, No 6,November-December 2015:742-753.

4. J. Lindstrom, J. Tuomilehto, The diabetes risk score: a practical tool to predict type 2 diabetes risk, Diabetes Care 26 (2003) 725-731.

5. Pablo Aschner M., Óscar Mauricio Muñoz V., Diana Girón C., Luz Ángela Casas., Olga Milena García., Daniel Fernández., et al., Colombian Clinical Practice Guideline for Treatment of Type 2 Diabetes Mellitus: First Line Treatment Failure. Universitas Médica 2017; 58(4)

6. Vargas H, Casas L. A, Epidemiología de la diabetes mellitus en Sudamérica: la experiencia de Colombia. Clin Investig Arterioscler. 2016: 28(5):245-256 http:// dx.doi.org/10.1016/j.arteri.2015.12.002 